\title{
REVIEW OF SINGLE POINT CUTTING TOOL GRINDER
}

\author{
S. L. Bankar ${ }^{1}$, Apekshit Sonole ${ }^{2}$ and Shivam Dharmadhikari ${ }^{3}$, \\ ${ }^{1}$ Associate Professor, ${ }^{2,3} \mathrm{UG}$ Students \\ Department of Mechanical Engineering, Yeshwantrao Chavan College of Engineering, Nagpur \\ Communicating Author Email: \{²asonole@gmail.com\}
}

\begin{abstract}
While machining a job on a lathe machine, quality of work done is generally assessed by the finishing and accuracy of the completed work piece. The wear of tool and its re-sharpening is key to good surface finish and accuracy on the work piece. Tool re-sharpening is generally a manual process is performed at a traditional bench grinder which requires operator's skill and accuracy. However, even the skillful operator is unable to replicate the exact angle specified for the job resulting in improper tool structure, uneven stresses within tool, and inappropriate machining of the job. In addition to that it also consumes a certain period of time which is inefficient when considered for production purpose. Also, the various irregularities in the tool such as uneven surfaces, roughness or the composition of the tool has a great impact on the final product to be machined. Therefore, it becomes very important to have the accurate tool geometry to achieve the desired material removal from the workpiece.

Taking these problems into considerations, we have developed a Single Point Cutting Tool Grinder (SPCTG). The main aim of the device is to achieve proper tool geometry after re-grinding and also to reduce the time required to do so.
\end{abstract}

Keyword: Grinder tool, cutting tool, rake angel, machines

\section{Introduction}

Single Point Cutting Tool Grinder is a machine developed to overcome the existing limitations of manual labor [1-2]. It is a bridgeway between the traditional methods and the highly modernized machinery for that purpose[3]. Hence the model so developed provides easy and automated process to re-sharpen the cutting tool. As known that the tool wears out and hence successive grinding operation is to be performed[4]. Due to the heavy and continuous machining process the rate of tool wear increases. Therefore to maintain the shape and size and other dimensions of the cutting tool accurate grinding is the most basic and important requirement [5]. This can be achieved by a SPCTG with highest the highest precision and accuracy .For that purpose a machine is developed such that it eliminates all the existing limitations associated with the purpose of making this machine. To achieve these elements within a job various factors are considered such as, Stability of the machine, Operator skill and Effectiveness of tool used i.e. proper tool geometry according to the type of job [6]. The removal of the material from the prescribed job is done using a Single Point Cutting Tool (SPCT). Hence it is important to maintain the specified dimensions of the various parameters of the SPCT accurately. Tool geometry, is one of the key aspects for the proper and efficient working of the cutting tool in the lathe machine. The quality or finishing of the job can be defined by how well the tool geometry was maintained during the entire process along with various elements determining the specifications such as speed, feed and depth of cut [7-8].

The single point cutting tool has 7 parameters namely
i. $\quad$ Back rake angle $\left(0^{\circ}\right)$
ii. Side rake angle $\left(7^{\circ}\right)$
iii. End relief angle $\left(6^{\circ}\right)$

\section{iv. Side relief angle $\left(8^{\circ}\right)$ \\ v. End cutting edge angle $\left(15^{\circ}\right)$ \\ vi. Side cutting edge angle $\left(16^{\circ}\right)$ and. \\ vii. Nose radius $(0.8 \mathrm{~mm})$}

Therefore the cutting of the work piece depends upon these angles as they are an integral component of the cutting tool. Hence a thorough knowledge of the specifications of the tool helps in selection of it according to the requirement.

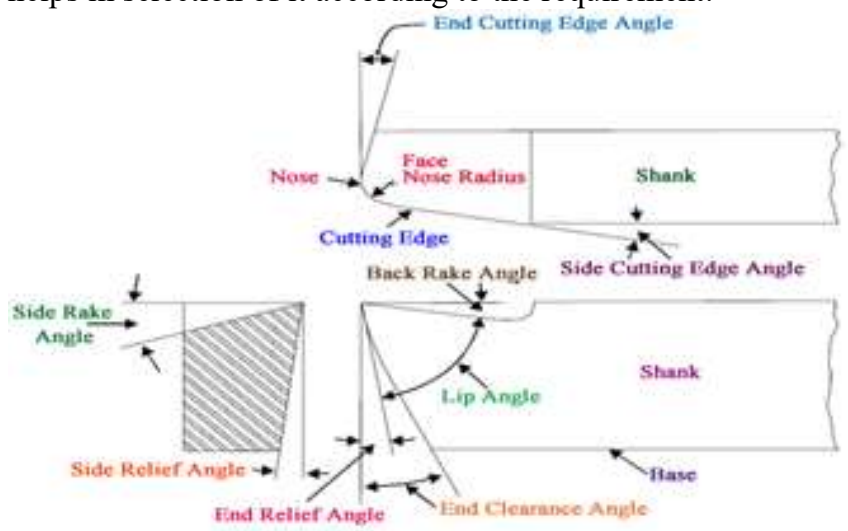

Fig. 1: Single point cutting tool.

\section{Review of Concept of Tool Wear}

It is determined as the restrained failure of cutting tool due to repetitive usage and machining operations. The effects of tool wear include blunt tips, and inaccurate geometry. General tools which are affected are ; tipped tools, drill bits, tool bits, etc.

Some widespread effects of tools wear are listed below:

i. Increase in cutting forces 

ii. Increase in cutting temperatures
iii. substandard surface finish
iv. decrease in accuracy of finished part
v. May cause tool breakage
vi. Can be leading to change in tool geometry

\section{Selection of Machining Parameters}

\subsection{Rake angle}

Rake angle is used to describe the angle between the cutting face and tool relative to the work. It is used to determine various machining parameters. Mainly there are three types of rake angles, they are : positive rake, zero rake or neutral, and negative rake angle.

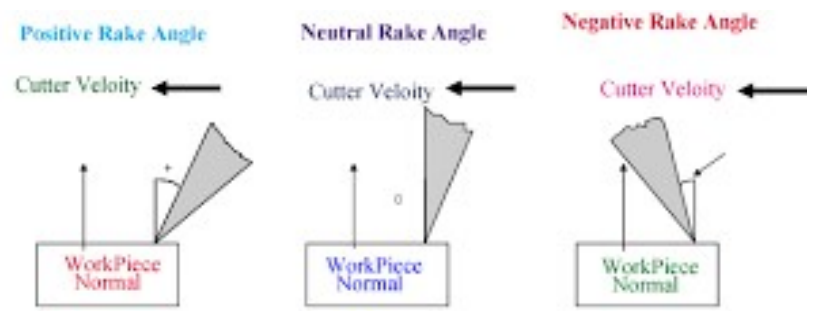

Fig. 2: Various types of Rake Angles

\subsection{Side Cutting Edge Angle}

The side cutting edge angle has a significant impact on effects of the back force, chip thickness and amount of feed force. The side cutting edge angle is also known as LEAD ANGLE.
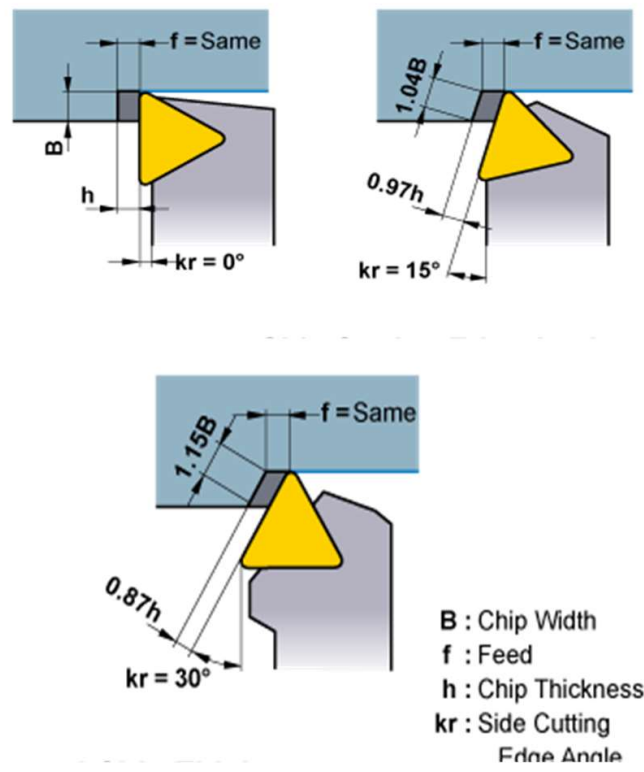

Fig. 3: Effects of side cutting edge angle

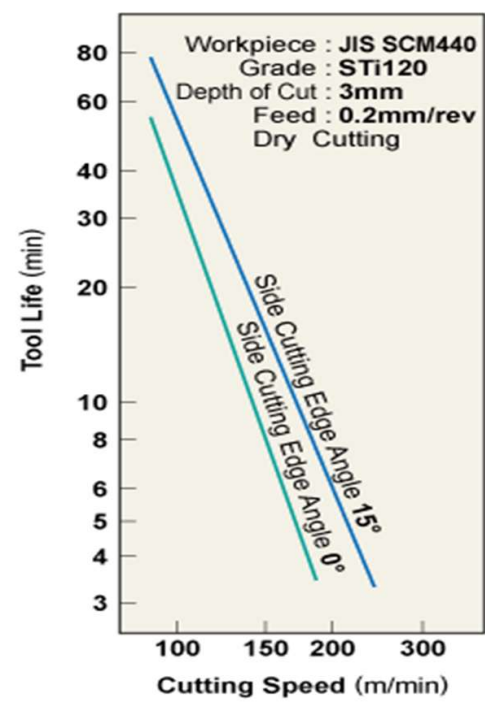

Fig. 4: Effect of tool life vs. cutting speed

i. At the similar feed rate, while increasing the side cutting edge angle, the chip contact length decreases chip thickness. As a result, the cutting force is effected on a larger cutting edge and tool life is increased significantly.

ii. Increasing the side cutting edge angle results in the increase of force a'. Thus, bending occurs in thin and long work pieces.

iii. While the side cutting edge angle is increased the chip control decreases simultaneously.

iv. Increasing the side cutting edge angle results in decreasing chip thickness and increase in chip width. Thus, breaking of chips is a difficult method.

\subsection{End Cutting Edge Angle}

End cutting edge angle is the angle between the end cutting edge and a line perpendicular to the shank of the tool is shown in.

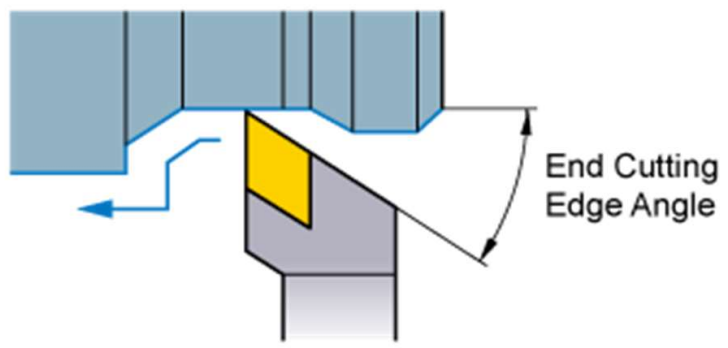

Fig. 5: Pictorial view of end cutting edge angle 


\section{Selection of Components}

A. Grinding Wheel

4 inch grinding wheel made of abrasive materials. It is very light in weight and causes less vibrations compared to others in the same category.

\section{B. 775 DC Supply Motor}

Operating range: $7 \mathrm{v}-14 \mathrm{v}$

Nominal voltage: $12 \mathrm{v}$

Maximum Speed: $6000 \mathrm{rpm}$

Current req: $5.25 \mathrm{~A}$

Torque req: $725 \mathrm{~g}-\mathrm{cm}$

\section{Mounting and Arbor}

The attachment is meant to connect a DC motor with a $5 \mathrm{~mm}$ shaft. It is useful to create DIY cutting/grinding/buffing tools, based on high-torque motors (typically RS-775 12V DC motor). The mandrel adaptor fits snugly on the motor shaft and there are two Allen key-based screws for additional fastening. The adaptor has a standard dimension and accommodates all forms of commonly available cutting/grinding discs with $16 \mathrm{~mm}$ bore.

\section{Principle of Working}

The main aim of the device is to achieve accurate tool geometry with presion and accuracy. In order to achieve it following arrangements were made.

Construction of grinders assembly is shown in Fig. 6. A metal based framework is made with the device mounted upon it for rigidity and reduced vibrations during working.

The feed mechanism is such that a feed base with a lead screw is taken for slow and steady feed, in accordance with the ongoing process of grinding the tool with infeed as well as outwards. The feed base is provided with a adjustable angular movement with ability to constrain itself according to a specific angle (Turn table). A restricter is also used, to ensure that the $\mathrm{JIG}$ is positioned correctly for grinding of the tool. The tool is fixed in a JIG, on a projected assembly with feature of tightening it with accordance to the user (via HT Bolt). The complete assembly as shown in Fig. 7. is mounted on a rail type structure where it is vertically constrained and allowed lateral movement.

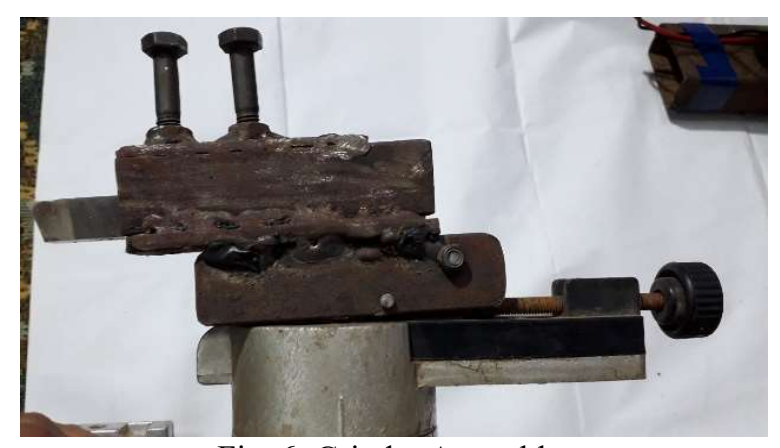

Fig. 6: Grinder Assembly.

All the three motors (Dc motor, 775) are mounted on the base structure. A complete isolation is ensured for rotation of all the grinding wheels. An Arbor (M10 Arbor) is employed for fixing of grinding wheel on the motor. The mounting of motor is such that it allows the grinding wheel to rotate in a defined angle preset by user. The angle can be varied can be varied with respect to the grinding requirement of Single point cutting tool.

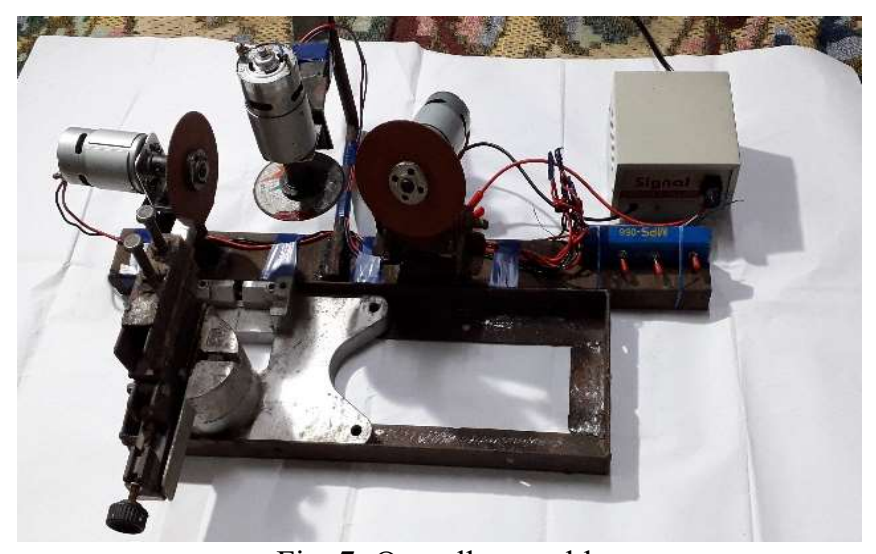

Fig. 7: Overall assembly

The blunt tool to be resharpened is mounted upon the JIG via Bolts, The feed base is then moved towards position 1 where SIDE CUTTING ANGLE is resharpened. The lead screw is rotated clockwise slowly and the tool is fed to the grinder for resharpening, it is then further retracted by rotating the screw anti-clockwise. The feed base is then moved to 2 nd position where END CUTTING ANGLE is addressed for resharpening and then moved to 3rd position for resharpening on TOP RAKE ANGLE.

A similar in-feed and retraction process must be followed to ensure proper grinding of the cutting tool. After the grinding of all the angles, the tool is removed from the JIG and can be used for lathe work on machine. 


\section{Conclusion and Discussions}

The resulting model can perform the task of grinding the various required angles of the grinder. The precision of the End Cutting Edge Angle; Side Cutting Edge Angle and Back Rake Angle of single point cutting tool to improve the accuracy of its re-sharpening without the aid of a skilled worker, which otherwise would be needed to perform a similar operation traditionally on a bench grinder. Thereby it eliminates the human error from the process.

The conventional time required to re-sharpen a SPCT on the traditional system can be reduced to a great extent as discussed in the result. Therefore the project automizes the process of resharpening. An unskilled worker can also complete the process without much skill as know he can perform the operation without much of a problem.

As we know that any SPCT comes with a specific set of angles and to accommodate the different angles of different tools we have provided various degrees of freedom to the grinder itself so that it can be adjusted to the pre-existing angles of the SPCT . By doing this the machine becomes available to a number of SPCT's used in the industry.

As this is a secondary machining operation, the primary being the Lathe machining it saves the transit time between two successive Lathe machining operations, thereby improving the overall efficiency of the industry.

\section{References}

[1] Doshi, T., Dave, P., Deorukhkar, R., Dubey, A. and Lohar, S., "Specialized Automated Tool Grinding Machine", International Journal of Engineering Research and General Science Volume 4, Issue 2, March-April, 2016 pp: 499-506.

[2] .Roe, J.W., 1916. English and American tool builders. Yale University Press.

[3] Vesali Alireza and Tawakoli Taghi, Study on Hydrodynamic, "Pressure in Grinding Contact Zone", 14, 2014, pp. 13-18.

[4] T.S. Rajpathak, Geometric modeling of single point cutting tools for grinding and sharpening, M.Tech Thesis, IIT Kanpur, 1996

[5] Tandon, P., Gupta, P. and Dhande, S.G., 2003. Geometric modeling of single point cutting tool surfaces. The International Journal of Advanced Manufacturing Technology, 22(1-2), pp.101-111.

[6] Heineman, R.K., 1956. Cutting tool grinding fixture. U.S. Patent 2,730,846.

[7] US2, 365,759 LOUIS H. HOWE DEC-23-1942 (lathe and shaper tool bit grinding gauge)

[8] US2, 887,823 A. PASSAROTTI APRIL-3-1958 (Tool grinding jig) 